\title{
Medição da cortical óssea através de radiografia panorâmica para diagnóstico de osteopenia e osteoporose em mulheres
}

\author{
Measurement of the cortical bone by panoramic radiography for the diagnosis \\ of osteopenia and osteoporosis in women \\ Rodrigo Coelho Bezerra Menezes ${ }^{1}$ (D), Bruna Balbino de Paula² (D), Yamba Carla Lara Pereira ${ }^{3}$ (D)
}

\begin{abstract}
RESUMO
Modelo do estudo: Estudo de prevalência. Objetivo: Avaliar a prevalência de osteopenia e osteoporose diagnosticadas por radiografia panorâmica através de medidas qualitativas do córtex ósseo mandibular. Materiais e métodos: Foram utilizados o índice de Klemetti e o índice de Benson através de paquímetro digital. A análise descritiva dos dados foi realizada no Statistical Package for the Social Sciences (SPSS) V.20.0. Resultados: Das cinquenta radiografias panorâmicas avaliadas de mulheres com média de idade de 43,04 anos, 15 mulheres (30\%) foram diagnosticadas com osteopenia pelo índice de Klemetti, não havendo casos de osteoporose na amostra. Em relação ao índice de Benson, os resultados mostraram média de 4,24 mm (DP: 0,54), com valor mínimo de 3,30 e máximo de 6,10 mm. Conclusão: Nossos achados evidenciam a importância da radiografia panorâmica para o diagnóstico precoce de osteopenia e osteoporose. A radiografia panorâmica apresenta baixo custo e facilidade de uso pelos cirurgiões-dentistas, sendo um exame amplamente utilizado em diversas áreas da odontologia.
\end{abstract}

Palavras-chave: Osteoporose; Radiografia Panorâmica; Mulheres.

\begin{abstract}
Study design: Prevalence study. Aim: Evaluating the prevalence of osteopenia and osteoporosis diagnosed by panoramic radiography through qualitative measurements of the mandibular bone cortex. Materials and methods: The Klemetti index and the Benson index were used through a digital caliper. The descriptive analysis of the date was performed in the Statistical Package for the Social Sciences (SPSS) V.20.0. Results: Of the fifty panoramic radiographs evaluated from women with a mean age of 43.04 years, 15 women (30\%) were diagnosed with Klemetti's osteopenia, and there were no cases of osteoporosis in the sample. Regarding the Benson index, the results showed an average of $4.24 \mathrm{~mm}$ (SD: 0.54), with a minimum value of 3.30 and a maximum of $6.10 \mathrm{~mm}$. Conclusion: Our findings highlight the importance of panoramic radiography for the early diagnosis of osteopenia and osteoporosis. Panoramic radiography presents low cost and ease of use by dental surgeons, being an exam widely used in several areas of dentistry.
\end{abstract}

Keywords: Osteoporosis; Panoramic Radiography; Women.

1. Centro Universitário Luterano de Palmas, Palmas (TO), Brasil.

2. Universidade de São Paulo, Ribeirão Preto (SP), Brasil.

$\square$ Bruna Balbino de Paula. Avenida Bandeirantes, 3900 - Monte Alegre. CEP 14040-901. Ribeirão Preto (SP), Brasil. brunabalbino@hotmail.com | Recebido em: 06/05/2019 | Aprovado em: 08/11/2019 


\section{INTRODUÇÃO}

A osteoporose é definida como uma doença esquelética, sistêmica e progressiva, caracterizada por baixa massa óssea (osteopenia) e deterioração do tecido ósseo. Esta condição promove aumento da fragilidade óssea e suscetibilidade à fratura ${ }^{1}$. A osteoporose é considerada um problema de saúde pública associada ao envelhecimento ${ }^{2}$ e dor crônica ${ }^{3}$. Por se tratar de uma doença crônica e multifatorial, pode progredir silenciosamente por décadas até que seja diagnosticada, resultando em situações que podem afetar a qualidade de vida das pessoas ${ }^{4}$.

A osteoporose é uma condição que possui altas taxas de morbidade e mortalidade, sendo as mulheres as mais afetadas ${ }^{5}$. Na faixa etária acima de 35 anos, a densidade mineral óssea (DMO) em homens e mulheres diminui gradualmente. No entanto, essa desmineralização óssea ocorre mais rapidamente em mulheres, especialmente após a menopausa, sendo considerada três vezes mais comum entre mulheres do que homens ${ }^{1}$. A osteoporose não apresenta manifestações clínicas específicas até a primeira fratura. Portanto, a história clínica e o exame físico detalhado devem ser realizados em todos os pacientes para identificar fatores que possam contribuir para a perda óssea, bem como para avaliar fatores preditivos para futuras fraturas e descartar causas secundárias de osteoporose. Alguns fatores de risco provavelmente serão revertidos ${ }^{6}$.

O exame padrão-ouro entre os métodos de imagem para o diagnóstico e prevenção da osteoporose é a densitometria óssea ${ }^{7}$. No entanto, o alto custo e o difícil acesso da população a mesma podem dificultar o seu uso como método de triagem $^{8}$. Em contrapartida, a radiografia panorâmica é rotineiramente utilizada para pacientes idosos antes da colocação de implantes dentários e próteses totais em desdentados. Dessa forma, a radiografia panorâmica pode ser considerada um método diagnóstico para diagnóstico prévio de osteoporose ${ }^{9}$. Reforçando essas informações, a radiografia panorâmica é caracterizada pela possibilidade de uma visão global de todos os elementos dentários da maxila e mandíbula, bem como de todos os constituintes ósseos ${ }^{10}$.

Devido à alta prevalência de osteoporose e o baixo custo da radiografia panorâmica, é extrema- mente importante realizar trabalhos que possam fornecer o embasamento para medição do córtex ósseo mandibular e possível diagnóstico precoce para prevenção ou tratamento rápido da osteoporose $^{11}$. Tendo em vista essas informações, o objetivo do presente estudo foi avaliar a prevalência de osteoporose medida através da radiografia panorâmica em mulheres de uma população do sul do Pará, utilizando o índice de Klemetti e Benson.

\section{MATERIAL E MÉTODOS}

\section{Caracterização da amostra}

Foram utilizadas cinquenta radiografias panorâmicas de mulheres atendidas em uma clínica privada localizada no sul do Pará. As radiografias panorâmicas foram obtidas através do equipamento Prolinaxc (Planmecaoy, Helsinki, Finlândia) com sensor digital e a análise foi realizada no laboratório de radiologia do Centro Universitário Luterano de Palmas durante o primeiro semestre de 2018. Os critérios de exclusão foram radiografias panorâmicas de homens e crianças

\section{Aspectos de natureza ética}

O presente trabalho foi submetido ao Comitê de Ética em Pesquisa do Centro Universitário Luterano de Palmas para avaliação ética e foi aprovado sob o número 2.661.938.

\section{Delineamento da análise}

O índice cortical mandibular (ICM) foi utilizado para avaliar o diagnóstico de osteoporose de forma qualitativa, considerando qualitativamente a margem endosteal da cortical mandibular, classificando-a como: C1 (normal) quando lisa e aguda, C2 (osteopenia) quando apresenta defeitos semilunares e C3 (osteoporose) quando é porosa e a espessura cortical é reduzida (Figura 1). Os diferentes níveis corticais da base óssea mandibular foram considerados variáveis. 


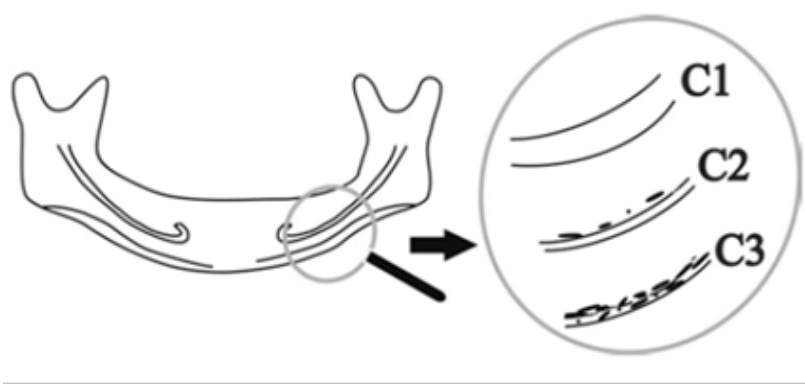

Figura 1: Índice mandibular cortical adaptado de Klemetti et al. $1994^{12}$.

A análise quantitativa foi baseada no índice panorâmico mandibular (IPM) proposto por Benson et al. $(1991)^{13}$, que estabelece a medida sobre a linha perpendicular a base da mandíbula, na altura do centro do forame mentual pela distância entre o limite inferior da base da mandíbula e seu limite máximo classificando em: cortical normal quando a espessura for maior ou igual a $3 \mathrm{~mm}$ e cortical com alteração quando o valor da espessura for menos que $3 \mathrm{~mm}$. Todas as medições foram realizadas avaliando a cortical óssea mandibular do lado direito do paciente, utilizando paquímetro digital.

\section{Análise estatística}

A análise descritiva dos dados foi realizada no Statistical Product Service Service (SPSS) V.20.0.

\section{RESULTADO E DISCUSSÃO}

No presente estudo, das 50 radiografias panorâmicas analisadas, a média de idade dos pacientes foi de 43,04 anos, sendo a idade mínima 21 anos e a idade máxima de 68 anos. A maior susceptibilidade de mulheres ao diagnóstico de osteopenia e osteoporose, principalmente durante a fase de pós-menopausa, já está bem estabelecida na literatura ${ }^{14-15}$. Dessa forma, estudo recente de análise da osteopenia e osteoporose em mulheres na pós-menopausa evidenciou que $55,9 \%$ das radiografias panorâmicas avaliadas apresentaram índice de cortical inferior ou igual a três (3) $\mathrm{mm}$, sendo que $90,9 \%$ dessa alteração óssea foi encon- trada em mulheres com mais de $70 \operatorname{anos}^{16}$. No presente estudo, foi evidenciada uma porcentagem menor de mulheres com índice cortical mandibular afetado, provavelmente devido a faixa etária da amostra como mostra a Tabela 1.

\section{Tabela 1}

Distribuição da amostra de acordo com a idade e o índice cortical mandibular.

\begin{tabular}{ccccccc}
\hline Idade & $20-30$ & $31-40$ & $41-50$ & $51-60$ & $61-70$ & Total \\
\hline$n$ & 8 & 13 & 14 & 11 & 4 & 50 \\
C1 & 8 & 13 & 14 & 0 & 0 & 35 \\
C2 & 0 & 0 & 0 & 11 & 4 & 15 \\
C3 & 0 & 0 & 0 & 0 & 0 & 0 \\
\hline
\end{tabular}

Além disso, evidenciamos que o índice de Benson apresentou uma média de 4,24 (Desvio Padrão, DP: 0,54 ), com mediana de 4,20. O valor mínimo foi de 3,30 e o máximo de 6,10. O intervalo de confiança de $95 \%$ para o limite inferior foi de 4,09 e o limite superior foi de 4,40. O percentil 25 foi de 3,87 e o percentil 75 foi de 4,52 . Vale ressaltar que é de suma importância o encaminhamento de pacientes para densitometria óssea cujas radiografias panorâmicas mostram extensas erosões na cortical inferior da borda mandibular ou espessura cortical abaixo de $3 \mathrm{~mm}$. Segundo Taguchi et al. (2006) ${ }^{17}$ a avaliação dos índices mental e mandibular cortical poderia predizer o diagnóstico de osteoporose e da baixa densidade mineral óssea em mulheres. No entanto, no presente estudo não foi evidenciado pacientes com espessura menor que $3 \mathrm{~mm}$.

$\mathrm{O}$ índice de Klemetti para o uso do diagnóstico de osteoporose por radiografias panorâmicas é uma ferramenta útil que pode ser utilizada por cirurgiões-dentistas na detecção de baixo mineral ósseo densidade ${ }^{10,12}$. Em estudo de Leite et al. (2008) com 351 mulheres, observou-se que aquelas classificadas como C3 tinham uma razão de chance de 5,16 vezes maior para o diagnóstico densitométrico de osteoporose. Ainda, em estudo de Bollen et al. $(2000)^{18}$ foi evidenciado que indivíduos que relataram fratura por osteoporose apresentavam a cortical mandibular com cerca de $0,54 \mathrm{~mm}$ mais fina. De fato, a osteoporose é um distúrbio osteometabólico que eleva a fragilidade óssea devido à perda de massa óssea e um desarranjo em sua microar- 
quitetura se agrava com a idade. As principais manifestações clínicas da osteoporose são as fraturas, sendo as mais frequentes as de vértebras, fêmur e ossos do antebraço ${ }^{19}$. Dessa forma, indivíduos com osteoporose têm mais reabsorção e redução da base da mandíbula cortical. Em contrapartida, indivíduos classificados com índices C1 e C2 têm grande chance de apresentar densitometria normal $^{20}$. Neste estudo, $70 \%$ das mulheres obtiveram o índice $\mathrm{C} 1,30 \%$ obtiveram o índice C2 e nenhum paciente apresentou índice C3.

A aplicação da radiografia panorâmica contribui para avaliação da porosidade do osso cortical e possibilita o diagnóstico precoce de fraturas. Neste contexto, foi evidenciado que mulheres pós-menopausadas com baixa densidade mineral óssea (DMO) do fêmur apresentam o córtex mandibular mais fino na região do forame mentual, bem como são mais suscetíveis a fraturas do colo ${ }^{21}$. Ainda, Delvin (2012) ${ }^{22}$ aponta que a espessura e as alterações morfológicas do osso cortical inferior mandibular estão associadas com a DMO, independentemente de idade, altura e peso. Além disso, Mahl et al. (2008) ${ }^{23}$ apontam que os índices panorâmicos mandibular, mental, mentoniano, antegoníaco e goníaco podem identificar baixa massa óssea no indivíduo. No entanto, apenas os índices panorâmicos mandibular e mental podem refletir pacientes com osteopenia/osteoporose ${ }^{24}$. Em conjunto, esses estudos demonstraram a relevância do uso de radiografias panorâmicas para o diagnóstico precoce da osteoporose, bem como a necessidade de disseminação e uso dessa ferramenta como auxílio no diagnóstico precoce da osteoporose, com base em estudos longitudinais e comparativos utilizando métodos padrão de ouro.

\section{CONCLUSÃO}

Nesse grupo de cinquenta mulheres com idade média abaixo da esperada para menopausa, não houve nenhum caso de osteoporose. No entanto, foi possível encontrar sinais de osteopenia. Dessa forma, nosso estudo corrobora a verificação inicial dos casos de alterações ósseas visíveis através das radiografias panorâmicas para posterior direcionamento dos pacientes para diagnóstico precoce de osteopenia e osteoporose.

\section{REFERÊNCIAS}

1. Kanis JA, Melton LJRD, Christiansen C, Johnston CC, Khaltaev N. The diagnosis of osteoporosis. J Bone Miner Res. 1994;9:1137-41.

2. Verheij JGC, Geraets WGM, Stelt PFV, Horner K, Lindh C, Nicopouloukarayianni K, et al. Prediction of osteoporosis with dental radiographs and age. Dentomaxillofac Radiol. 2009;38:431-7.

3. Paolucci T, Saraceni VM, Piccinini G. Management of chronic pain in osteoporosis: challenges and solutions. J Pain Res. 2016;9:177-86.

4. Ardakani FE, Mirmohamadi SJ. Osteoporosis and oral bone resorption: a review. J Maxillofac Oral Surg. 2009;8:121-6.

5. Burge R, Dawson-Hughes $B$, Solomon DH, Wong JB, King $A$, Tosteson A. Incidence and economic burden of osteoporosis-related fractures in the United States, 2005-2025. J Bone Miner Res. 2007;22(3):465-75.

6. Papaioannou A, Morin S, Cheung AM, Atkinson $S$, Brown JP, Feldman S, ET AL. Clinical practice guidelines for the diagnosis and management of osteoporosis in Canada. CMAJ. 2010;182:1864-73.

7. Meunier PJ, Delmas PD, Eastell R, Mcclung MR, Papapoulos $\mathrm{S}$, Rizzoli R, et al. Diagnosis and management of osteoporosis in postmenopausal women: clinical guidelines. International Committee for Osteoporosis Clinical Guidelines. Clin Ther. 1999;21(6):1025-44

8. Costa-paiva L, Horoviz A, Santos A, Fonsechi-Carvasan GA, Pinto-Neto AM. Prevalência da osteoporose em muIheres na pós-menopausa e associação com fatores clínicos e reprodutivos. RBGO. 2003;25(7):507-12.

9. Leite AF, Figueiredo PTS, Guia CM, Melo NS, Paula AP. Radiografia Panorâmica - Instrumento Auxiliar no Diagnóstico da Osteoporose. Rev Bras Reumatol. 2008;48(4):226-33.

10. Calciolari E, Donos N, Park JC, Patrie A, Mardas N. Panoramic Measures for Oral Bone Mass in Detecting Osteoporosis: A Systematic Review and Meta-Analysis. JDR Clinical Research Supplement. 2015:255-75.

11. Kim OS, Shin MH, Song IH, Lim IG, Yoon SJ, Kim OJ, et al. Digital panoramic radiographs are useful for diagnosis of osteoporosis in Korean postmenopausal women. John Wiley \& Sons A/S and The Gerodontology Association. Published by John Wiley \& Sons Ltd. Gerodontology, 2014; p 7-8,

12. Klemetti E, Kolmakov S, Kroger H. Pantomography in assessment of the osteoporosis risk group. Scand J Dent Res. 1994;102(1):68-72.

13. Benson BW, Prihoda TJ, GLASS BJ. Variations in adult cortical bone mass as measured by a panoramic mandibular index. Oral Surg Oral Med Oral Pathol. 1991;71(3):349-56.

14. Paschalis EP, Fratzl P, Gamsjaeger S, Hassler N, Brozek W, Eriksen EF, et al. Aging Versus Postmenopausal Osteoporosis: Bone Composition and Maturation Kinetics at Actively-Forming Trabecular Surfaces of Female Subjects Aged 1 to 84 Years. J Bone Miner Res. 2016;31(2):347-57. 
15. Balaguer JR, Olmos JM. Prevalence of osteopenia and osteoporosis in postmenopausal women. Aten Primaria. $2005 ; 35(7): 342-5$.

16. Camargo AJ, Arita ES, Fernández MCC, Watanabe PCA. Comparación de dos métodos radiológicos para evaluación de densidad ósea en mujeres posmenopáusicas. Int J Morphol. 2015; 33(2):732-6.

17 Taguchi A, Tsuda M, Ohtsuka M, Kodama I, Sanada M, Nakamoto T, et al. Use of dental panoramic radiographs in identifying younger postmenopausal women with osteoporosis. Osteoporos Int. 2006;17(3):387-94.

18. Bollen AM, Taguchi A, HUJOEL PP, Hollender LG. Case-control study on selfreported osteoporotic fractures and mandibular cortical bone. Oral Surg Oral Med Oral Pathol Oral Radiol Endod. 2000;90(4):518-24.

19. Lindsay R, Silverman SL, Cooper C, Hanley DA, Barton I, Broy $S B$, et al. Risk of new vertebral fracture in the year following a fracture. JAMA. 2001;17(3):320-3.
20. Halling A, Persson GR, Berglund J, Johansson O, Renvert $\mathrm{S}$. Comparison between the Klemetti index and heel DXA BMD measurements in the diagnosis of reduced skeletal bone mineral density in the elderly. Osteoporos Int. 2005;16(8):999-1003.

21. Horner K, Allen PD, Graham J, Jacobs R, Boonen S, Pavitt $\mathrm{S}$, et al. The relationship between the OSTEODENT index and hip fracture risk assessment using FRAX. Oral Surg Oral Med Oral Pathol Oral Radiol Endod. 2010;110:243-9.

22. Delvin H. Identification of the Risk for Osteoporosis in Dental Patients. Dent Clin North Am. 2012;56(4):847-61.

23. Mahl CRW, Licks R, Fontanella VRC. Comparação de índices morfométricos obtidos na radiografia odontológica panorâmica na identificação de indivíduos com osteoporose/osteopenia. Radiol Bras 2008. 41(3):183-7.

24. Kowalchuk RM, Dalinka MK. The radiologic assessment of osteoporosis. Orthop J. 1998;11:67-72. 